\title{
Understanding the physics of DNA using nanoscale single- molecule manipulation
}

\author{
Eric W. Frey, Ashton A. Gooding, Sitara Wijeratne, and Ching-Hwa Kiang ${ }^{\dagger}$ \\ Department of Physics and Astronomy, Rice University, Houston, TX 77005, USA
}

\begin{abstract}
Processes for decoding the genetic information in cells, including transcription, replication, recombination and repair, involve the deformation of DNA from its equilibrium structures such as bending, stretching, twisting, and unzipping of the double helix. Single-molecule manipulation techniques have made it possible to control DNA conformation and simultaneously detect the induced changes, revealing a rich variety of mechanically-induced conformational changes and thermodynamic states. These single-molecule techniques helped us to reveal the physics of DNA and the processes involved in the passing on of the genetic code.
\end{abstract}

\section{Keywords}

single-molecule manipulation; the physics of DNA

\section{Introduction}

DNA is the carrier of genetic information and is involved in biomolecular processes such as transcription and replication. Many of these processes are governed by the mechanics and thermodynamics of bending, stretching, twisting, and unzipping the double helix [1-6]. Double-stranded DNA (dsDNA) is a semi-flexible polymer, with its base-stacking architecture and negative charges along its phosphate backbone. In physiological conditions, thermal fluctuations do not bend it significantly on length scales below $50 \mathrm{~nm}$, which is equivalent to 150 base pairs (bp) [7]. The $10 \mu \mathrm{m}$-long DNA of a viral genome can be packed inside a capsid of $50 \mathrm{~nm}$ in diameter [8-10], and in eukaryotic cells, histones bend DNA into loops of $10 \mathrm{~nm}$ in diameter. The latter serves as the first step in the hierarchical packaging of the genome in eukaryotes (Fig. 1), and it regulates gene expression by obstructing access to base pairs [11]. Histones, helicases, topoisomerases, and RNA and DNA polymerases are examples of proteins that generate or relieve tension and torque in DNA to enable its biochemical functions [5, 12-15]. With advances in single-molecule techniques, it has been possible to examine the physics of DNA directly. By providing control and measurement of force of a single molecule, these techniques have revealed a variety of DNA conformations and much of DNA's complex behavior.

\section{Single-molecule manipulation experiments}

Single-molecule manipulation techniques using atomic force microscopy (AFM), optical tweezers, and magnetic tweezers are illustrated in Fig. 2. These techniques have been used to manipulate a variety of biological molecules. In each of these methods, a single DNA molecule is attached between a substrate and a force probe, either an AFM tip or a micron-

\footnotetext{
(c) Higher Education Press and Springer-Verlag Berlin Heidelberg 2012

†chkiang@ rice.edu.
} 
sized bead, in an aqueous solution. The change in molecular end-to-end distance is determined from the change in probe and substrate positions. The force on the molecule is determined from displacement of the probe relative to its equilibrium position. Nonspecific attachment, typically used in AFM, is achieved by adsorption of DNA to the substrate surface or the probe surface. Specific attachment, employed by optical and magnetic tweezers, is achieved by functionalization of probe and substrate surfaces. These modifications exploit the high affinity and specificity of binding among ligand-receptor, antibody-antigen pairs and DNA hybridization. Other techniques elongate DNA by confining the molecule within micro or nano-sized obstacles. Such techniques include driving DNA electrophoretically through microlithographic arrays [17], nanochannels [18, 19], and nanopores [20].

In AFM [Fig. 2(a)], the force probe is an AFM tip attached to a cantilever, and the substrate is mounted on a piezoelectric scanner. Moving the substrate toward the AFM tip allows nonspecific or specific molecular attachment between the substrate and cantilever. When the molecule is attached to the tip and the substrate, moving the substrate away from the cantilever produces force on the attached molecule, and the resulting bending of the cantilever is detected by the deflection of the laser beam reflecting off the back of the cantilever onto a position-sensitive detector. The force exerted on the molecule, $F$, is determined by Hooke's law, $F=-K z$, where $K$ is the cantilever's spring constant and $z$ is the cantilever displacement from its equilibrium position. Using the equipartition theorem,

the spring constant is determined using $\frac{1}{2} k_{\mathrm{B}} T=\frac{1}{2} K\left\langle z^{2}\right\rangle$, where $k_{\mathrm{B}}$ is Boltzmann's constant and $T$ is temperature [23]. AFM cantilevers used for single-molecule manipulation typically have a spring constant $K=10 \mathrm{pN} / \mathrm{nm}$ or higher. This results in unloaded cantilever fluctuations of at least $5 \mathrm{pN}$ at room temperature, which sets the limit of the noise level in the force on an attached molecule measurable by AFM. AFM is able to measure high forces up to a few nanoNewtons, the limit usually being set by the strength of the attachment [24-26].

In a typical optical tweezers setup [Fig. 2(b)], the force probe is a micrometer-sized dielectric bead captured in an optical trap. The substrate may be the side of a translatable fluid chamber or a second bead held by either a micropipette or a second optical trap $[4,12$. 14]. The optical trap consists of a tightly-focused laser, which exerts a three-dimensional restoring force on a dielectric bead trapped near the laser focus. To minimize photo-damage to the trapped biomolecules, near-infrared wavelengths are used [27-29]. The displacement of the bead from the trap center can be measured by video tracking via an optical microscope. For small displacements of the bead, the force is determined using Hooke's law, and the trap stiffness is determined using the equipartition theorem, as in the case of AFM. Optical traps typically have spring constants ranging from $0.005-1 \mathrm{pN} / \mathrm{nm}$, which is smaller than the AFM cantilevers. The low noise level allows measurement of forces on the molecule as low as $0.1 \mathrm{pN}$. Optical tweezers are generally used to probe forces less than 100 $\mathrm{pN}$, where the ligand-receptor or antibody-antigen pairs used to attach the DNA unbind [30].

Magnetic tweezers [Fig. 2(c)] are similar to optical tweezers, except that the force probe consists of a super-paramagnetic bead in an applied magnetic field. The force on the bead is proportional to the gradient of the square of the magnetic field. In addition, a torque is applied to the bead due to its small magnetic polarization anisotropy, which tends to align the bead with the applied magnetic field. Thus, by rotating the applied field, the attached molecule can be twisted as well as stretched [31,32]. Magnetic tweezers have miniscule stiffness as low as $10^{-6} \mathrm{pN} / \mathrm{nm}$, allowing them to probe forces as low as $10^{-3} \mathrm{pN}$. Like optical tweezers, they can probe up to $100 \mathrm{pN}$ until the DNA handles break [33-35]. 
Each of these single-molecule manipulation techniques has advantages and disadvantages. While AFM is capable of probing large forces up to a few nanoNewtons, it is generally not sensitive for probing forces less than $5 \mathrm{pN}$. Optical tweezers and magnetic tweezers, on the other hand, can measure forces as small as $0.1 \mathrm{pN}$ and $10^{-3} \mathrm{pN}$, respectively. However, these techniques have an upper limit for the forces that can be studied, since the DNA handles usually break around $100 \mathrm{pN}$. Other considerations include spatial and temporal resolution, and stability and drift control. For high spatial resolution, subnanometer precision is routinely obtained using AFM [36], and optical tweezers has achieved high spatial resolution in a recent work [37]. Video tracking of beads in optical tweezers and magnetic tweezers offers a simple method for measuring position, but with less resolution (around $5 \mathrm{~nm}$ ) and sampling rate limited to $500 \mathrm{~Hz}$ for fast cameras [38], while greater resolution and sampling rates are possible using more sophisticated methods [39]. Dynamic position control is implemented in AFM and optical tweezers using a piezoelectric scanner with capacitive position detection in a feedback loop, with an accuracy of $1 \mathrm{~nm}$ or better. This enables force-clamping, a mode in which the extension of the molecule is measured over time while maintaining a constant applied force. Magnetic tweezers are uniquely suited to high-bandwidth force-clamping at low cost, without the need for sophisticated feedback loops, since large magnet displacements change the applied force only slightly (typically 1 $\mathrm{pN}$ for a displacement of $1 \mathrm{~mm}$ ).

Recent efforts have been made to combine fluorescence imaging with single-molecule manipulation [40, 41]. For example, by using fluorescent dyes which bind to specific conformations of DNA, this combination of methods can distinguish different structures of DNA under applied force [42]. In addition, fluorescence can be used to pinpoint the location where an applied force has the largest effect on DNA [43]. Finally, the large forces obtainable in an AFM experiment enabled direct fluorescence imaging of DNA strand rupture [44].

\section{Polymer physics models of DNA}

Single-molecule manipulation experiments measure the force-extension curve of DNA, and the data are fitted to polymer physics models to determine parameters that define its mechanical properties (Fig. 3). In solution, DNA adopts a random coil conformation which minimizes free energy. Extending the molecule imposes a constraint limiting the number of accessible conformations, thus the work done on the molecule is mainly used to offset the reduced entropy. For dsDNA, at forces less than $10 \mathrm{pN}$ the force-extension curve is dominated by this entropic elasticity. At higher forces, dsDNA begins to exceed its contour length and, consequently, its double-helix structure is disrupted. The polymer elasticity models which best describe the force-extension curves of single-stranded DNA (ssDNA) and dsDNA are the freely-jointed chain (FJC) and wormlike chain (WLC) models, respectively.

In the FJC model, the polymer consists of a chain of freely rotating segments of characteristic Kuhn length. The extensible FJC assumes that the polymer is stretchable, and the force is related to extension $x$ by $[30,46]$

$$
x=b_{s s}\left[\operatorname{coth}\left(2 \beta P_{s S} F\right)-\frac{1}{2 \beta P_{S S} F}\right]\left(1+\frac{F}{K_{s S}}\right)
$$

where $P_{s s}, b_{s s}$, and $K_{s S}$ are the persistence length, contour length, and stretch modulus of ssDNA, respectively, and $\beta=1 /\left(k_{\mathrm{B}} T\right)$. The persistence length is a measure of bending stiffness. The $K_{S S}$ accounts for the extensibility of the molecule. For ssDNA, $P_{s S}=0.75 \mathrm{~nm}$ and $K_{s S}=800 \mathrm{pN}$ [30, 47-49]. 
The WLC models a polymer as a flexible rod characterized by a bending stiffness. In an extensible WLC model, force can be related to extension by $[45,50]$

$$
x=b_{d s}\left(1-\frac{1}{\sqrt{4 \beta P_{d s} F}}+\frac{F}{K_{d s}}\right)
$$

where $P_{d s}, b_{d s}$, and $K_{d s}$ are the persistence length, contour length, and elastic stretch modulus of dsDNA, respectively. For dsDNA, $P_{d s}=50 \mathrm{~nm}$ and $K_{d s}=1200 \mathrm{pN}$ [30, 47-49].

\section{The overstretching transitions and force-induced melting}

Figure 4(a) is a typical force-extension curve of dsDNA. At low forces, the molecule behaves like a WLC. When the force reaches $65 \mathrm{pN}$, the force-extension curve shows a plateau, indicating a cooperative transition of the molecular conformation [30, 51]. Recent experiments demonstrate that both melting and a B-DNA to S-DNA transition occurs at this force, depending on environmental factors [52-54]. At forces around $150 \mathrm{pN}$, dsDNA melts into ssDNA, where the force-extension curve is best described by the extensible FJC model [Eq. (1)] with a persistence length and stretch modulus consistent with ssDNA [30, 47, 48]. Another example is poly(dA) (ssDNA composed only of adenine bases), where distinct plateaus and multiple force-extension pathways have been observed [21, 55]. One poly(dA) pathway is similar to that of random-sequence ssDNA, whereas the other pathway has an additional, energetically favored transition [Fig. 4(b)], possibly to a state in which the bases are weakly stacked. The multiple pathways suggest that poly(dA) has two conformational states when stretched close to twice its contour length. Further study is required to elucidate the structures responsible for these transitions and whether they can be tuned by changing environmental conditions, such as salt concentration and temperature.

Pulling single DNA molecules has been found to unzip as well as stretch DNA. Unzipping occurs when the secondary structure, i.e., the double helix of dsDNA is disrupted, resulting in unpairing of the bases. The dynamics of unzipping are sequence-dependent, as evidenced by higher observed forces in GC-rich regions [56] and good reproducibility for unzipping/ rezipping molecules of the same sequence $[57,58]$.

\section{Conclusion}

The response of the DNA molecule to a force is crucial in many biochemical functions, including transcription, replication, recombination, and packaging. Fundamental DNA properties are being revealed as melting and complex reactions are being probed. Understanding quantitatively the detailed mechanical property of single- and doublestranded DNA allows us to characterize different DNA conformational states and their associated energetics. This quantitative information will help us predict DNA behavior and interactions that are important in biological and medical systems.

\section{Acknowledgments}

We thank NSF DMR-0907676, Welch Foundation No. C-1632, Hamill Innovation Fund, NIH/NIBIB T32EB009379-03, and the Alliance for NanoHealth, NASA/DOE DE-FG02-08ER64712 for support.

\section{References}

1. Hogan ME, Austin RH. Nature. 1987; 329(6136):263. [PubMed: 3627268]

2. Goodman SD, Nash HA. Nature. 1989; 341(6239):251. [PubMed: 2528697]

3. Stasiak A, Capua ED. Nature. 1982; 299(5879):185. [PubMed: 7050731] 
4. Hegner M, Smith SB, Bustamante C. Proc Natl Acad Sci USA. 1999; 96(18):10109. [PubMed: 10468570]

5. Johnson DS, Bai L, Smith BY, Patel SS, Wang MD. Cell. 2007; 129(7):1299. [PubMed: 17604719]

6. Bloomfield, VA.; Crothers, DM.; Tinoco, I, Jr. Nucleic Acids: Structures, Properties, and Functions. Sausalito, California: University Science Books; 2000.

7. Baumann CG, Smith SB, Bloomfield VA, Bustamante C. Proc Natl Acad Sci USA. 1997; 94(12): 6185. [PubMed: 9177192]

8. Smith DE, Tans SJ, Smith SB, Grimes S, Anderson DL, Bustamante C. Nature. 2001; 413(6857): 748. [PubMed: 11607035]

9. Fuller DN, Raymer DM, Kottadiel VI, Rao VB, Smith DE. Proc Natl Acad Sci USA. 2007; 104(43): 16868. [PubMed: 17942694]

10. Fuller DN, Rickgauer JP, Jardine PJ, Grimes S, Anderson DL, Smith DE. Proc Natl Acad Sci USA. 2007; 104(27):11245. [PubMed: 17556543]

11. Felsenfeld G, Groudine M. Nature. 2003; 421(6921):448. [PubMed: 12540921]

12. Brower-Toland BD, Smith CL, Yeh RC, Lis JT, Peterson CL, Wang MD. Proc Natl Acad Sci USA. 2002; 99(4):1960. [PubMed: 11854495]

13. Strick TR, Croquette V, Bensimon D. Nature. 2000; 404(6780):901. [PubMed: 10786800]

14. Abbondanzieri EA, Greenleaf WJ, Shaevitz JW, Landick R, Block SM. Nature. 2005; 438(7067): 460. [PubMed: 16284617]

15. Wuite GJL, Smith SB, Young M, Keller D, Bustamante C. Nature. 2000; 404(6773):103. [PubMed: 10716452]

16. Luger K, Mäder AW, Richmond RK, Sargent DF, Richmond TJ. Nature. 1997; 389(6648):251. [PubMed: 9305837]

17. Volkmuth WD, Austin RH. Nature. 1992; 358(6387):600. [PubMed: 1501715]

18. Tegenfeldt JO, Prinz C, Cao H, Chou S, Reisner WW, Riehn R, Wang YM, Cox EC, Sturm JC, Silberzan P, Austin RH. Proc Natl Acad Sci USA. 2004; 101(30):10979. [PubMed: 15252203]

19. Reisner W, Morton KJ, Riehn R, Wang YM, Yu Z, Rosen M, Sturm JC, Chou SY, Frey E, Austin RH. Phys Rev Lett. 2005; 94(19):196101. [PubMed: 16090189]

20. Sauer-Budge AF, Nyamwanda JA, Lubensky DK, Branton D. Phys Rev Lett. 2003; 90(23): 238101. [PubMed: 12857290]

21. Chen W-S, Chen W-H, Chen Z, Gooding AA, Lin K-J, Kiang C-H. Phys Rev Lett. 2010; 105(21): 218104. [PubMed: 21231359]

22. Lionnet T, Joubaud S, Lavery R, Bensimon D, Croquette V. Phys Rev Lett. 2006; 96(17):178102. [PubMed: 16712339]

23. Hutter JL, Bechhoefer J. Rev Sci Instrum. 1993; 64(7):1868.

24. Rief M, Clausen-Schaumann H, Gaub HE. Nat Struct Biol. 1999; 6(4):346. [PubMed: 10201403]

25. Staple DB, Loparic M, Kreuzer HJ, Kreplak L. Phys Rev Lett. 2009; 102(12):128302. [PubMed: 19392329]

26. Lv S, Dudek DM, Cao Y, Balamurali MM, Gosline J, Li H. Nature. 2010; 465(7294):69. [PubMed: 20445626]

27. Neuman KC, Chadd EH, Liou GF, Bergman K, Block SM. Biophys J. 1999; 77(5):2856. [PubMed: 10545383]

28. Koirala D, Dhakal S, Ashbridge B, Sannohe Y, Rodriguez R, Sugiyama H, Balasubramanian S, Mao H. Nat Chem. 2011; 3(10):782. [PubMed: 21941250]

29. Gibaud T, Barry E, Zakhary MJ, Henglin M, Ward A, Yang Y, Berciu C, Oldenbourg R, Hagan MF, Nicastro D, Meyer RB, Dogic Z. Nature. 2012; 481(7381):348. [PubMed: 22217941]

30. Smith SB, Cui YJ, Bustamante C. Science. 1996; 271(5250):795. [PubMed: 8628994]

31. Strick T, Allemand J, Croquette V, Bensimon D. Prog Biophys Mol Biol. 2000; 74(1-2):115. [PubMed: 11106809]

32. Lipfert J, Wiggin M, Kerssemakers JW, Pedaci F, Dekker NH. Nat Commun. 2011; 2:439. [PubMed: 21863006] 
33. Strick TR, Allemand J-F, Bensimon D, Croquette V. Biophys J. 1998; 74(4):2016. [PubMed: 9545060]

34. Yan J, Skoko D, Marko JF. Phys Rev E. 2004; 70(1) 011905.

35. Chen H, Fu H, Zhu X, Cong P, Nakamura F, Yan J. Biophys J. 2011; 100(2):517. [PubMed: 21244848]

36. Welker J, Giessibl FJ. Science. 2012; 336(6080):444. [PubMed: 22539715]

37. Stigler J, Ziegler F, Gieseke A, Gebhardt JCM, Rief M. Science. 2011; 334(6055):512. [PubMed: 22034433]

38. Bérut A, Arakelyan A, Petrosyan A, Ciliberto S, Dillenschneider R, Lutz E. Nature. 2012; 483(7388):187. [PubMed: 22398556]

39. Shank EA, Cecconi C, Dill JW, Marqusee S, Bustamante C. Nature. 2010; 465(7298):637. [PubMed: 20495548]

40. del Rio A, Perez-Jimenez R, Liu R, Roca-Cusachs P, Fernandez JM, Sheetz MP. Science. 2009; 323(5914):638. [PubMed: 19179532]

41. Deng Y, Sun M, Shaevitz JW. Phys Rev Lett. 2011; 107(15):158101. [PubMed: 22107320]

42. van Mameren J, Gross P, Farge G, Hooijman P, Modesti M, Falkenberg M, Wuite GJL, Peterman EJG. Proc Natl Acad Sci USA. 2009; 106(43):18231. [PubMed: 19841258]

43. Lang MJ, Fordyce PM, Engh AM, Neuman KC, Block SM. Nat Methods. 2004; 1(2):1.

44. Hards A, Zhou C, Seitz M, Bräuchle C, Zumbusch A. Chem Phys Chem. 2005; 6(3):534. [PubMed: 15799480]

45. Marko JF, Siggia ED. Macromolecules. 1995; 28(26):8759.

46. Wang MD, Yin H, Landick R, Gelles J, Block SM. Biophys J. 1997; 72(3):1335. [PubMed: 9138579]

47. Clausen-Schaumann H, Rief M, Tolksdorf C, Gaub HE. Biophys J. 2000; 78(4):1997. [PubMed: 10733978]

48. Cocco S, Yan J, Léger J-F, Chatenay D, Marko J. Phys Rev E. 2004; 70(1) 011910.

49. Calderon CP, Chen W-H, Lin K-J, Harris NC, Kiang C-H. J Phys: Condens Matter. 2009; 21(3) 034114.

50. Odijk T. Macromolecules. 1995; 28(20):7016.

51. Cluzel P, Lebrun A, Heller C, Lavery R, Viovy J-L, Chatenay D, Caron F. Science. 1996; 271(5250):792. [PubMed: 8628993]

52. Fu H, Chen H, Marko JF, Yan J. Nucleic Acids Res. 2010; 38(16):5594. [PubMed: 20435680]

53. Fu H, Chen H, Zhang X, Qu Y, Marko JF, Yan J. Nucleic Acids Res. 2011; 39(8):3473. [PubMed: 21177651]

54. Zhang X, Chen H, Fu H, Doyle PS, Yan J. Proc Natl Acad Sci USA. 2012; 109:8103. [PubMed: 22532662]

55. Ke C-H, Humeniuk M, S-Gracz H, Marszalek PE. Phys Rev Lett. 2007; 99(1) 018302.

56. Essevaz-Roulet B, Bockelmann U, Heslot F. Proc Natl Acad Sci USA. 1997; 94(22):11935. [PubMed: 9342340]

57. Danilowicz C, Coljee VW, Bouzigues C, Lubensky DK, Nelson DR, Prentiss M. Proc Natl Acad Sci USA. 2003; 100(4):1694. [PubMed: 12574500]

58. Hatch K, Danilowicz C, Coljee V, Prentiss M. Phys Rev E. 2007; 75(5) 051908. 
(a) Slort region of DNA double helix.

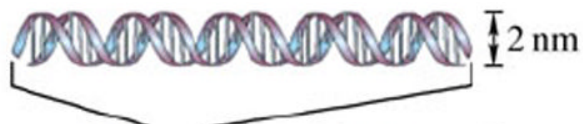

"Beads on a string" form of chromatin

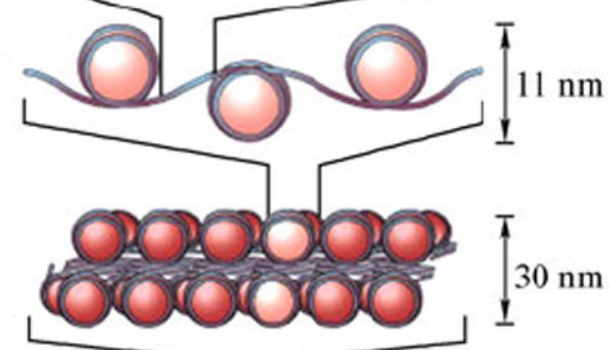

30-nm chromatin fibre of packed nucleosomes

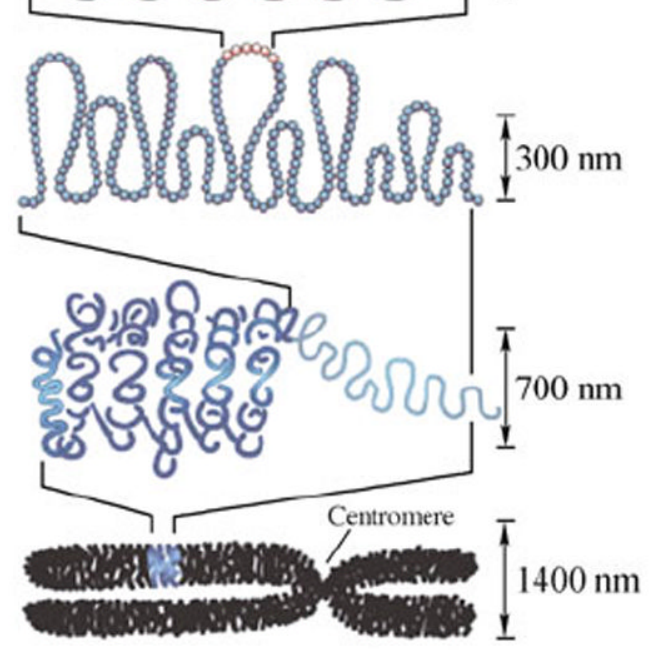

Section of chromosome in an extended form chromosome

(b)

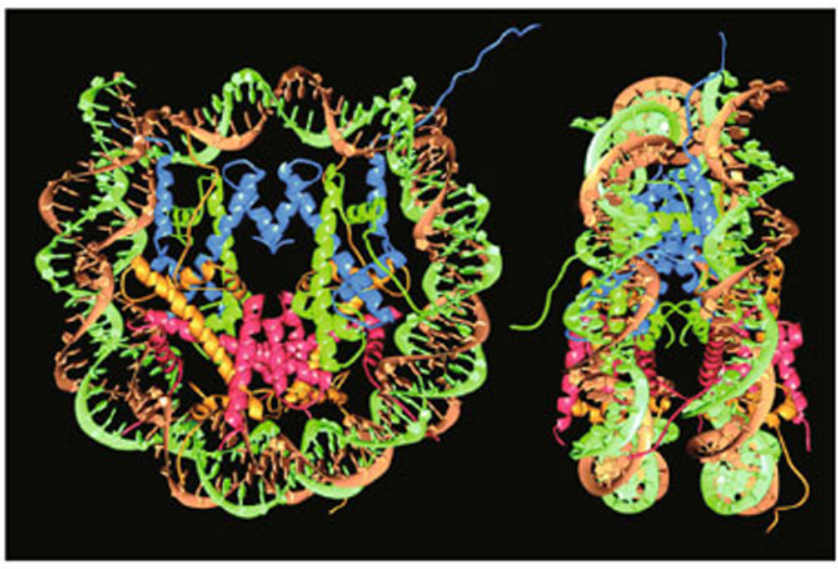

Fig. 1.

(a) Hierarchical organization of DNA packaged into a chromosome. The nucleosomes are formed by histones which bend DNA into small loops. Reproduced from Ref. [11], Copyright (๑) 2003 Nature Publishing Group. (b) Structure of the nucleosome. Two superhelical turns of the DNA double helix wrap around an octamer of histones through hydrogen bonds and electrostatic interactions. Reproduced from Ref. [16], Copyright $(0$ 1997 Nature Publishing Group. 
(a)

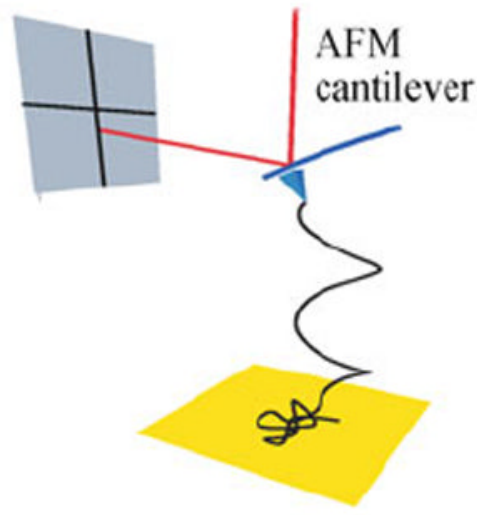

(b)

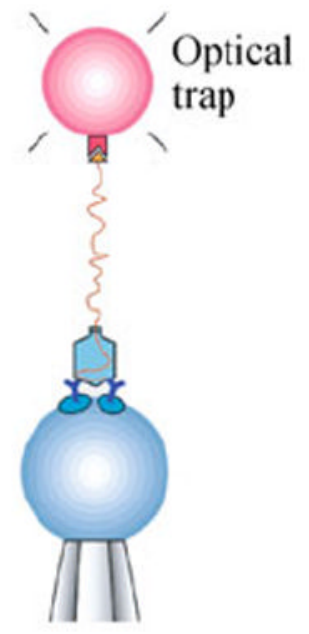

(c)

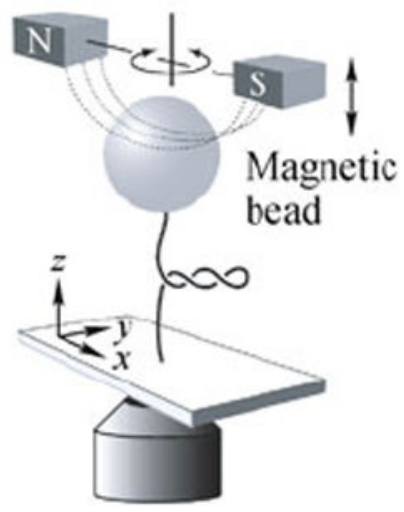

Fig. 2.

Illustrations of single-molecule manipulation techniques. (a) AFM. The molecule is held by the tip and the substrate surface. The force on the attached molecule is determined based on the deflection of the cantilever. Reproduced from Ref. [21], Copyright ( 2010 American Physical Society. (b) Optical tweezers. One end of a DNA molecule is attached to a bead trapped by a laser beam, while the other end is attached to a DNA-virus capsid complex on a second bead, held by a micropipette tip. Reproduced from Ref. [8], Copyright $\odot 2001$ Nature Publishing Group. (c) Magnetic tweezers. A force is exerted on the molecule by an attached super-paramagnetic bead in a magnetic field. The molecule can be twisted as well as stretched by the applied field. Reproduced from Ref. [22], Copyright () 2006 American Physical Society. 
(a)
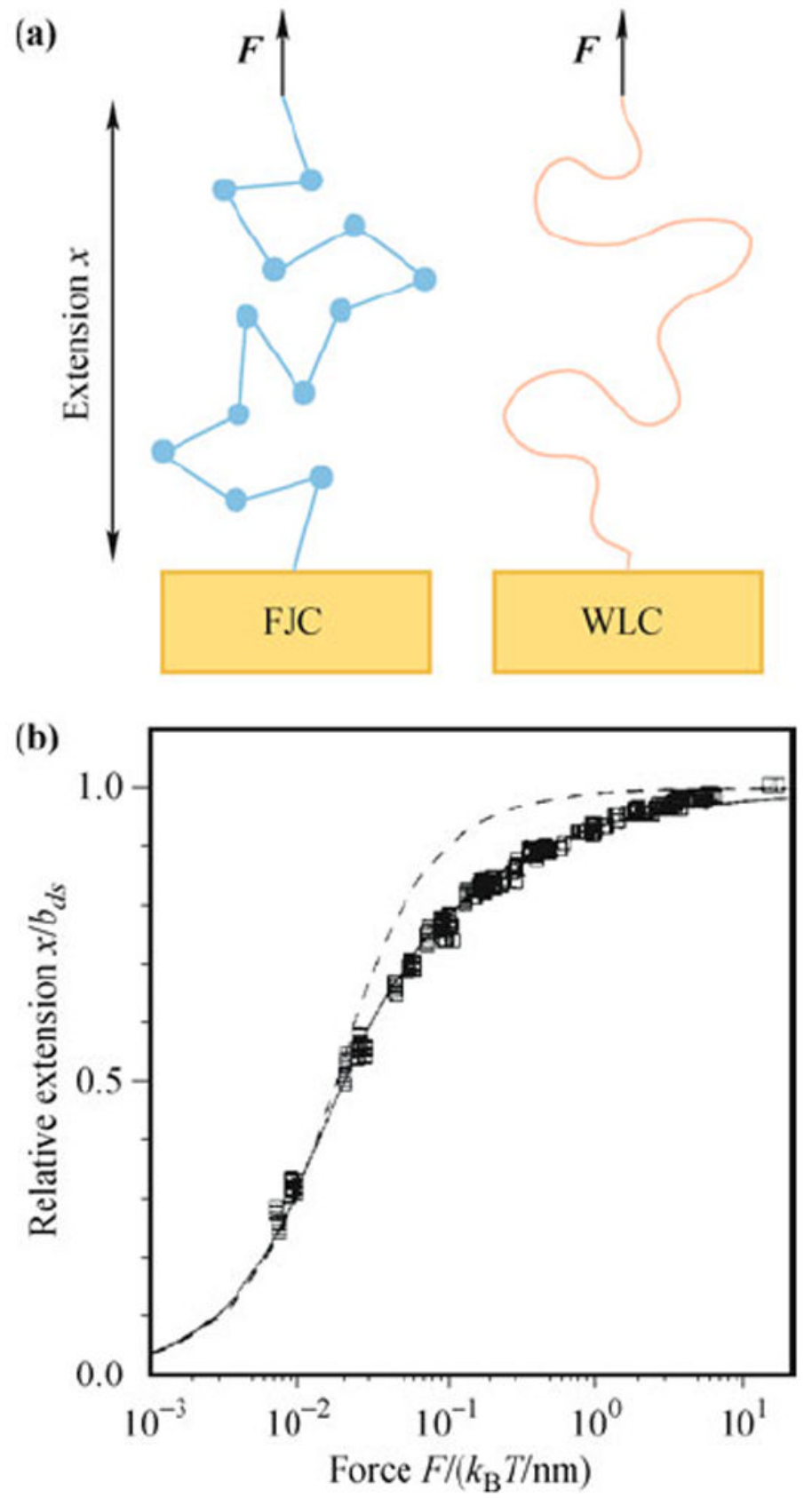

Fig. 3.

The polymer physics models that describe DNA. (a) Illustration of the FJC and WLC models. (b) Force-extension behavior of a single dsDNA molecule. dsDNA can be described accurately by the WLC model (solid curve), but not the FJC model (dashed curve). Reproduced from Ref. [45], Copyright @ 1995 American Chemical Society. 

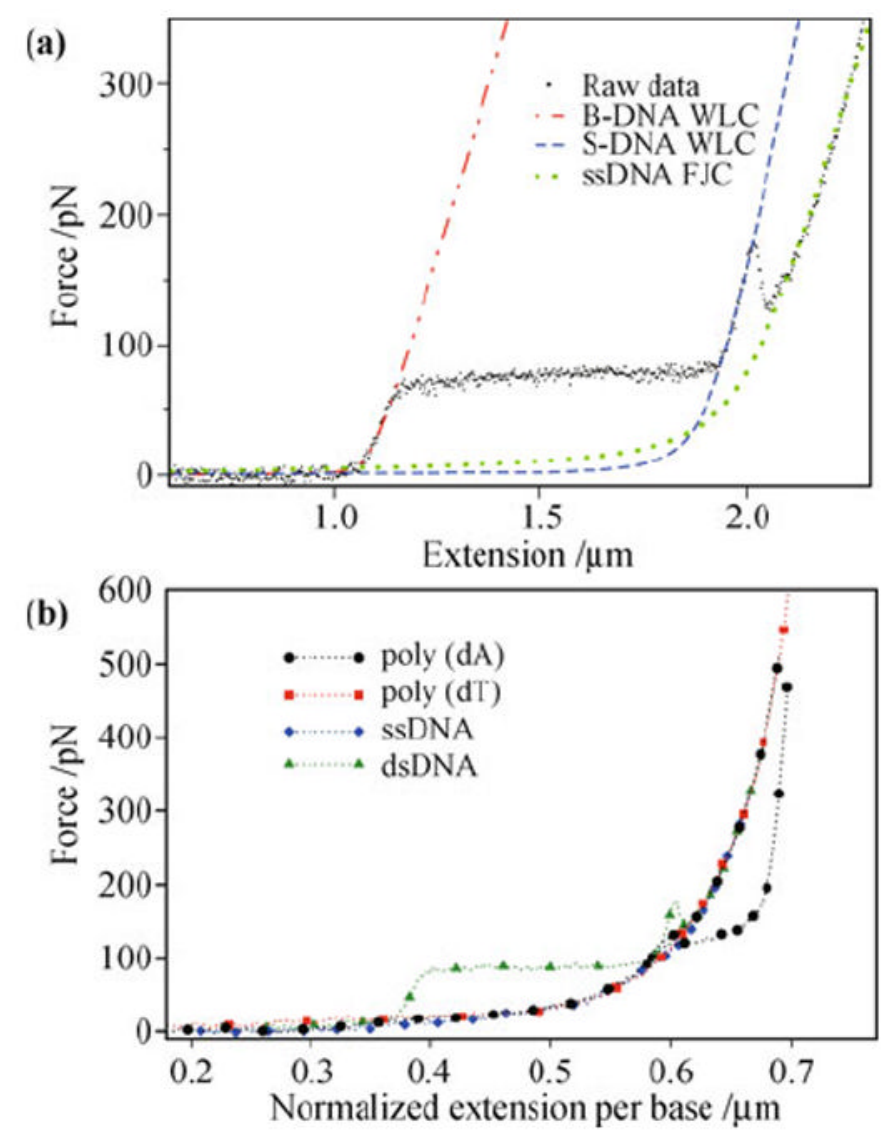

Fig. 4.

Force-induced transitions of DNA. (a) Force-extension data showing stretching, melting, and overstretching of a $\lambda$-DNA. The data are fit for extensible WLC and FJC models. Reproduced from Ref. [49], Copyright (C) 2009 IOP Publishing. (b) Force-extension pathways for poly(dA) compared to dsDNA and other ssDNA. Reproduced from Ref. [21], Copyright (C) 2010 American Physical Society. 